\section{L'erreur historique du corps médical}

\author{
J.-M. Guinchard
}

Disons le tout net: le pouvoir politique, à quelque niveau qu'il se situe, n'a jamais vraiment aimé les disciples d'Esculape. Depuis les Chamans jusqu'au druides gaulois, en passant par les «hommes médecine" des indiens et les sorciers africains, le pouvoir exécutif a toujours eu une position ambivalente visà-vis du rôle de la médecine ou de celui qui était considéré comme tel au sein de la cellule politique de base que celle-ci soit représentée par le village, la communauté, la famille au sens large ou la tribu.

Si le pouvoir exécutif était donné aux plus forts ou à celui choisi ou élu par ses pairs, le pouvoir du guérisseur ou du sorcier était quasi de droit divin. Cette dualité à la tête de la communauté posait dès l'abord le problème de concurrence entre le pouvoir temporel et le pouvoir spirituel. De plus, le sorcier étant quasi détenteur d'un pouvoir de vie et de mort, il concurrençait très sérieusement le pouvoir politique.

De tout temps, jusqu'à ces récentes années, il donc été dans les objectifs du pouvoir politique ou temporel de réduire cette concurrence.

Sans remonter aussi loin que nous pourrions le souhaiter, nous nous limiterons donc à examiner les actions du pouvoir politique et ses incidences sur la vie et la survie du corps médical durant le vingtième siècle. Au début de celui-ci, la décision a été prise d'instituer une assurance maladie, non obligatoire mais dont les affiliés pouvaient, moyennant cotisation, bénéficier de soins dispensés par des médecins reconnus ou ayant accepté de travailler à charge d'un assureur social, cette acceptation ayant pour conséquence un empiètement sur l'exercice libéral de notre art et un sacrifice au niveau de la tarification. Au lendemain de la guerre, le pouvoir politique s'est aperçu que les médecins n'étaient pas assez nombreux et que de ce fait, pouvaient tenir la dragée haute et constituaient un contre pouvoir important, ce d'autant plus qu'il détenait le savoir en matières de soins. Il a donc été décidé d'en former de plus en plus afin qu'une pléthore savamment organisée permette, et nous y sommes aujourd'hui, de réduire singulièrement le

Correspondance:

Dr J.-M. Guinchard

Association des Médecins du Canton de Genève

12, rue Micheli-du-Crest

Case postale 665

CH-1211 Genève 4 pouvoir des médecins et de leur imposer toute une série de contingences.

La troisième étape, celle dont nous vivions cruellement les conséquences, a été d'abord de rendre la loi sur l'assurance maladie obligatoire et d'autre part d'étendre au maximum le catalogue des prestations qu'elle fournissait. Cela a fait l'objet de la votation sur la LAMal, entrée en vigueur en 1996. Son extension obligatoire à tout un chacun avait, il faut le souligner, l'indéniable avantage de supprimer les réserves et de faciliter ainsi les passages d'une assurance à l'autre. Toutefois, en en étendant massivement le catalogue des prestations, on parvenait habilement à éviter que le médecin ne facture quoi que ce soit en dehors de ce qui était strictement prévu par la LAMal. A preuve, l'activité d'un médecin généraliste, d'un interniste, d'un pédiatre ou d'un psychiatre, tous médecins de premier recours, ne se conçoit plus en dehors de la LAMal et ces médecins se retrouvent donc à l'heure actuelle quasi salariés d'un assureur social pour plus de 95\% de leur activité journalière.

L'on pourra nous rétorquer que ces trois étapes sont le fruit du hasard et qu'elles n'ont pas été voulues sciemment par le pouvoir politique. Nous sommes d'un autre avis et même si nous ne prêtons pas toujours de grandes qualités d'anticipation au pouvoir politique, nous sommes persuadés qu'en l'état, c'est bien ce but qui a été poursuivi afin d'en arriver à la situation actuelle.

L'erreur historique du corps médical a été d'accepter de commencer à facturer à charge d'un assureur social. Cette erreur était toutefois compréhensible puisque le médecin avait, à cette époque et de cette façon, la quasi-certitude d'être remboursé pour son activité, certitude qui lui échappait parfois lorsqu'il facturait dans un régime privé à des patients qui n'avaient pas forcément les moyens de s'acquitter de leurs prestations. C'est là aussi toute l'ambivalence du médecin qui s'érige farouchement en tenant d'une médecine dite libérale mais qui, d'un autre côté, cherche par tous les moyens à voir son activité honorée financièrement et sans risque. Or, le risque de ne pas être payé fait partie intégrante de la qualité libérale de quiconque exerce une profession de ce type.

Erreur historique il y a donc eu, il s'agit maintenant de savoir non pas comment la réparer, mais comment s'adapter à un environnement nouveau. Un sage a dit un jour - il s'agissait, je crois, de Confucius - qu'il ne fallait pas éviter les erreurs, parce qu'elles nous apportaient les expériences les plus fructueuses. C'est vrai, mais il faut en tirer les conséquences afin de prendre d'autres options.

La seule option possible est l'anticipation sur une situation à venir. En matière d'assurances sociales, il ne nous reste plus une marge énorme, puisqu'il sera bien difficile à l'avenir de diminuer le catalogue de prestations consenti par la LAMal. A tout le moins peut-on espérer que tout médecin qui aurait une nouvelle prestation à mettre sur le marché saura éviter qu'elle soit à tout prix prise en charge par l'assureur social et la facturera à titre privé comme une prestation hors du catalogue LAMal. 
Les seules possibilités d'anticipation qui nous restent, le sont sur le plan des assurances privées. On sait que celles-ci ont fortement diminué avec la hausse des primes, et que nombre de patients trop âgés ou considérés comme représentant un trop grand risque, ont vu leur primes augmenter d'une façon si abrupte qu'ils ont été obligés de renoncer à leur assurance.

A l'heure actuelle, ce secteur devient également un objet de combat de la part des assureurs qui pratiquent en même temps l'assurance sociale et l'assurance privée. Désireux de contenir les coûts et de préserver leurs bénéfices dans un secteur où ceux-ci sont encore juteux, certains assureurs commencent à fixer des plafonds à leurs remboursements sans en avertir formellement ni les médecins ni les assurés ou peutêtre par le biais de conditions générales dont personne ne s'inflige habituellement la lecture fastidieuse et parfois peu compréhensible.

D'autres assureurs, souhaitant préserver l'existence des assurances privées et continuer à pouvoir les offrir à des tarifs abordables, approchent des associations médicales cantonales afin de conclure avec elles des conventions portant sur la garantie de remboursement d'une prestation jusqu'à un certain niveau.

Contrairement à ce qu'on pourrait penser, il ne s'agit pas là d'un "diktat» étatique puisque émanant du privé, mais bien d'une solution visant à garantir à l'assuré le remboursement d'une prestation dont les montants auront été librement discutés entre partenaires d'un même secteur.

Nous voyons là une mesure idéale d'anticipation, mesure qui permettrait au corps médical de défendre la qualité de ses prestations et, subsidiairement, le prix qu'il y attache en étant certain que ces montants là seront bel et bien remboursés aux assurés, sans que ne se pose ensuite des problèmes de recours de l'assuré contre le médecin ou de refus de paiement d'une différence lourde à supporter entre le montant demandé par le médecin et celui remboursé par l'assureur.

Nous pensons très sincèrement que s'offre à nous dans ces circonstances une possibilité, non plus de simplement réagir, mais très clairement de pro-agir en gardant la maîtrise d'une négociation qui, sans notre participation, va purement et simplement nous échapper. Des exemples récents avec le Groupe Mutuel ont montré que, sans aucun scrupule, un assureur pouvait parfaitement unilatéralement décider de la quantité remboursable de ces prestations en assurance maladie privée. Vaut-il donc mieux que cette quantité soit décidée par un seul assureur ou en commun entre membres du corps médical et assureurs privés qui connaissent tous deux la branche dans laquelle ils exercent. Poser la question, c'est y répondre et permettre d'éviter qu'une erreur historique commise au début de ce siècle n'ait des répercussions dans un secteur qui, pour l'instant demeure encore gérable par les médecins. 